\title{
Enhancing the Visualization of Laue Diffraction for Analyzing Strength Model Parameters
} \author{
Cynthia Bolmell, Kyle Ramos**, and Darby Jon Luscher ${ }^{\dagger}$ \\ ${ }^{*}$ CCS-7, Los Alamos National Laboratory, Los Alamos, USA \\ E-mail: ayan@lanl.gov, ahrens@lanl.gov \\ ${ }^{\dagger} \mathrm{T}-3$, Los Alamos National Laboratory, Los Alamos, USA \\ E-mail: djwalters@lanl.gov, dj1@lanl.gov \\ ${ }^{\ddagger}$ CCS-6, Los Alamos National Laboratory, Los Alamos, USA \\ E-mail: dfrancom@lanl.gov, earl@lanl.gov \\ $\S$ P-23, Los Alamos National Laboratory, Los Alamos, USA \\ E-mail: sjue@lanl.gov \\ T-1, Los Alamos National Laboratory, Los Alamos, USA \\ E-mail: jlbarber@lanl.gov \\ ${ }$ M-9, Los Alamos National Laboratory, Los Alamos, USA \\ E-mail: cbolme@lanl.gov \\ **M-7, Los Alamos National Laboratory, Los Alamos, USA \\ E-mail:kramos@lanl.gov
}

Ayan Biswas*, David Walters ${ }^{\dagger}$, Devin Francom ${ }^{\ddagger}$, Sky Sjue $^{\S}$, Earl Lawrence ${ }^{\ddagger}$, John L. Barber`, James Ahrens*,

\begin{abstract}
X-ray diffraction is important for analyzing crystals and their structures. Since the simulated diffraction patterns are costly, surrogate models are developed to enable fast time-tosolution. However, surrogate models produce slightly different output from the ground truth (simulation). Understanding and exploring these differences easily is an important topic for materials scientists. Visual comparison of these differences is difficult because diffraction spots are often very sparse and occupy few pixels in the images. In this work, we propose to use Voronoi-based tessellations to enhance visualizations of diffraction patterns. We propose to use this method for exploration of individual images as well as for visualizing the differences between the emulated and simulated outputs. We use Cinemabased viewers for exploration of the analyzed output. Domain expert feedback is provided to validate the utility of the proposed visualization and exploration system.
\end{abstract}

Index Terms-Bragg spots, visual exploration, interactive, difference visualization

\section{INTRODUCTION}

$\mathrm{X}$-ray diffraction is a method for evaluating the atomic or molecular structure of crystalline solids. The resulting diffraction patterns encode information related to crystalline lattice structure (size and shape). Further information such as deformation, changes in temperature, and residual stress is elucidated when comparing multiple diffraction patterns imaged during or following physical changes to the sample.

Studying diffraction patterns on powdered samples (those containing several pieces of crystalline solids oriented randomly throughout the sample) is well established since these random orientations produce Debye-Scherrer rings (see Fig 1) . Using powder diffraction, the radii of the rings provide a measure of the lattice spacings present in the sample. The width of the rings give an indication of both crystal size and residual stress in the rings. Under dynamic experiments, changes in the rings' radii can be attributed to elastic strain in the crystal and/or thermal expansion. Broadening of the rings along with a decrease in the intensity can indicate an increase in temperature or melting. Appearance of new rings or disappearance of existing rings can indicate phase change.

However, X-ray diffraction on a single crystalline lattice produces a more sparse dataset, albeit with the same characteristics described previously encoded in the pattern. A comparison of the difference between single crystal diffraction and powder diffraction is illustrated in Fig. 1 using sugar.

Single crystal diffraction is particularly useful for evaluating the mechanical properties of highly anisotropic crystalline materials. In-situ measurements of the stress and strain of a dynamically loaded material are useful for calibrating new physics based strength models [1]. In particular, the explosive crystal RDX ( $\alpha$-cyclotrimethylene Trinitramine) has been imaged using X-Ray diffraction at the Advanced Photon Source at Argonne National Laboratory for use with calibrating the strength model described by Luscher et al. [1]. In order to calibrate these models using experimental data, many simulations of the experiment must be performed to find the optimal model parameters which reproduce the X-ray diffraction pattern.

To speed this process up, emulators are used as a surrogate to the simulation to quickly probe the space of possible pa- 

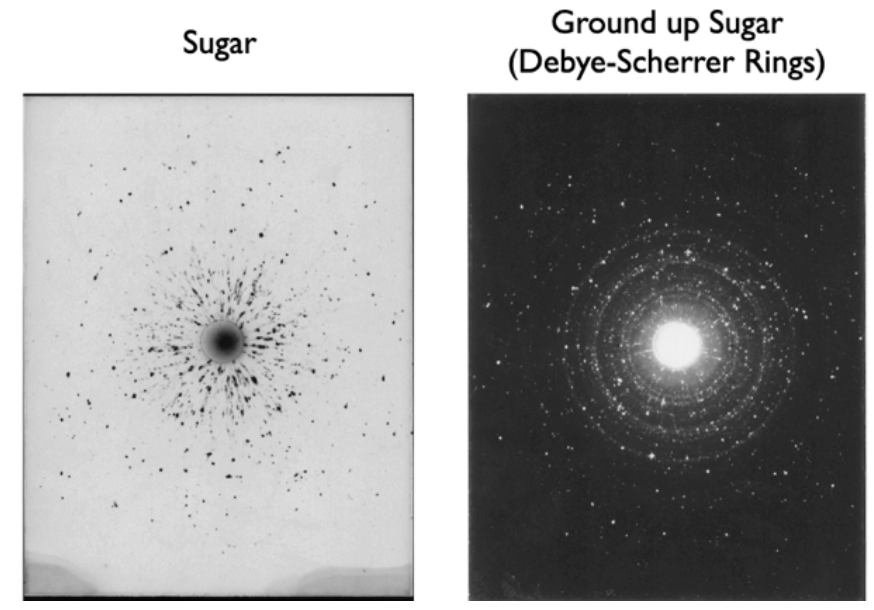

Fig. 1. (Left)Laue pattern of spots (black) for a single crystal of sugar. (Right) Debye-Scherrer rings produced from diffraction through ground (polycrystalline) sugar. (Photo credits: University of Cincinnati College of Engineering)

rameters and their predicted diffraction patterns. An emulator is a statistical or machine learning approximation to a computationally intensive simulation. It is built using a parsimonious training set of input-output pairs from the simulation. For a choice of simulation inputs, the emulator can make predictions of the simulation output. The prediction is approximate, but orders of magnitude faster than the simulation. This makes the emulator a viable tool for Bayesian inference or optimization procedures to learn which inputs make the simulator match observed experimental outputs. These procedures can sometimes require millions of evaluations, which would not be feasible with the simulator itself. This speed also makes emulators a useful tool for scientific visualization.

However, this presents a new challenge. Is the emulator good enough to produce diffraction patterns that are similar enough to those produced by simulations? What is the best way to visualize and quantify differences between comparative diffraction patterns? In the future, do these new visualization methods enable linking diffraction pattern characteristics with specific model parameters?

In this work, we develop an interactive visualization tool to explore spatial patterns of Bragg spots from simulations of single crystal deformation [1]. The tool facilitates comparison of simulated and emulated output for these diffraction spots. Our proposed visualization tool enables easy identification of which parameter combinations produce higher/lower errors in the emulation process compared with the simulated results. We use Voronoi tessellation and Cinema-based viewers [3] to achieve the aforementioned goals. We have collected feedback from the domain experts that clearly indicates the utility of our proposed visualization workflow.

\section{BACKGROUND AND MOTIVATION}

$\mathrm{X}$-ray diffraction is an imaging technique which enables in-situ measurements of the structure of a material. X-rays

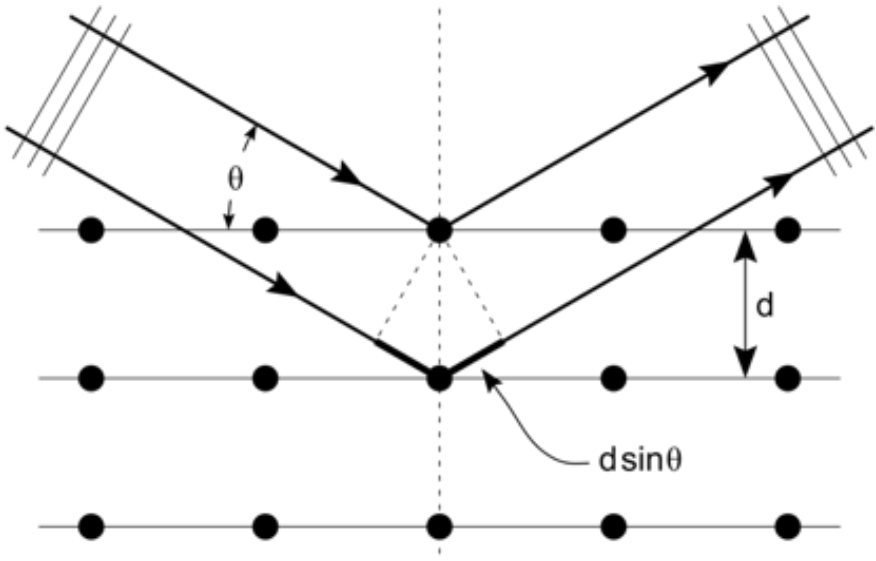

Fig. 2. Lattice spacing determines the angle of the diffraction peak.

interact with the electrons of an atom by scattering the Xrays in a process called elastic scattering: secondary spherical waves emanating from an atom. If the atomic or molecular structure of a material is ordered enough, the scattered waves will sync up in a pattern of constructive interference according to Bragg's law (illustration in Figure 2), that is:

$$
2 d \sin \theta=n \lambda
$$

Here, $d$ is the spacing between diffracting planes, $\theta$ is the incident and reflected angle, $n$ is any integer, and $\lambda$ is the wavelength of the incoming X-ray beam. The scattered Xrays that satisfy Bragg's law appear as spots on a detector positioned downstream from the sample. Incident X-ray beams often contain a spectrum of wavelengths causing a spatially varying field of constructive and destructive interference causing distinct patterns on X-ray detectors that are highly dependent on position.

Diffraction through a single crystal with a perfect crystalline lattice produces a sparse collection of spots on a detector called a Laue pattern. The lattice characteristics are encoded in the spatial distribution of spots. The process of interpreting these images becomes more complex with the use of polychromatic light (a spectrum of $\lambda$ ) and imperfect crystals. Small changes in incoming X-ray wavelengths, sample position, and detector positions can have highly discontinuous effects on the resulting diffraction image. However, single crystal diffraction has promise as an extremely informative diagnostic tool for parameterizing material models.

In an effort to understand and use X-ray diffraction as a diagnostic tool, we have begun recreating experiments using simulation and emulation tools to produce simulated X-ray diffraction patterns [4]. We are able to simulate the lattice structure of dynamically loaded crystalline solids using a Los Alamos National Laboratory research hydrocode called FLAG [2]. The deformed lattice information resulting from these simulations is used as to simulate the resulting X-ray diffraction patterns [4]. In our test case, we are analyzing the deformation of single crystals of the explosive RDX 


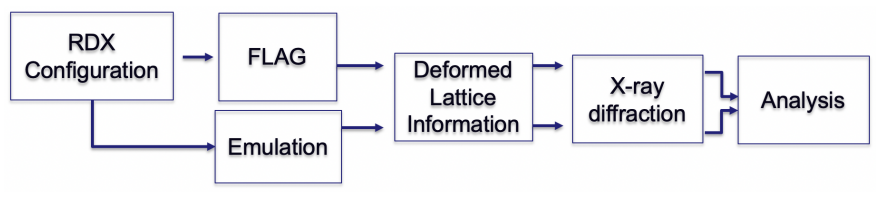

Fig. 3. Schematic representation of the data generation workflow.

\begin{tabular}{|c|c|c|c|c|c|c|}
\hline $\begin{array}{l}\text { Load } \\
\text { data }\end{array}$ & $\rightarrow$ Thresholding & $\begin{array}{c}\text { Apply } \\
\text { connected } \\
\text { components }\end{array}$ & $\longrightarrow$ & $\begin{array}{c}\text { Find } \\
\text { component } \\
\text { centers }\end{array}$ & $\rightarrow$ & $\begin{array}{l}\text { Voronoi } \\
\text { tessellation }\end{array}$ \\
\hline
\end{tabular}

Fig. 4. Schematic representation of our proposed method using Voronoi tessalation.

(cyclotrimethylene trinitramine). Using emulation tools, we are able to interpolate between model parameters to produce a full range of single crystal X-ray diffraction images, allowing for the exploration of model parameter sensitivities on the resulting diffraction image.

\section{OVERVIEW}

We produce both simulated (using FLAG [2]) and emulated (from our previously developed emulation tool [5], [6]) Bragg patterns for RDX using a common set of inputs parameter settings. Both simulation and emulation produce deformed lattice structures for each input setting and these are used to generate the $\mathrm{x}$-ray diffraction pattern for comparative analysis. $\mathrm{X}$-ray diffraction patterns are calculated by a LANL forwardscattering code known as BarberShop [4], which treats the deformation of each element in a FLAG simulation as an effective polycrystalline grain with a unique, perfect crystal structure. The shape of the array of Bragg spots from each such grain is calculated in 3D Fourier space, and their contribution to the complex scattered field on the detector is determined. Since the scattering patterns for different photon energies do not interfere with one another, the resulting intensities can simply be added to obtain the broadband pattern. Our data generation workflow is schematically presented in Figure 3.

For providing an enhanced visualization of the diffraction patterns, we primarily propose to use Voronoi tessellation. Starting from the diffraction patterns, we first threshold of data to identify the Bragg spot locations. Next, we identify the individual spots by using an algorithm to determine the connected pixels comprising a spot. Next, for each connected component, we identify the centers of these spots and use those spot centers as markers for the spot locations. Finally, we use these centers to tessellate the space. This workflow is presented in Figure 4. After the data processing, Cinema [3] is used for visualization purposes. More details of the workflow is discussed in the Method section(Section IV).

\section{METHOD}

The original premise was to compare diffraction patterns between a plate impact experiment on single crystal $\alpha$-RDX and several simulations of that experiment. Various strength model parameters (described in ref [1]) would be supplied

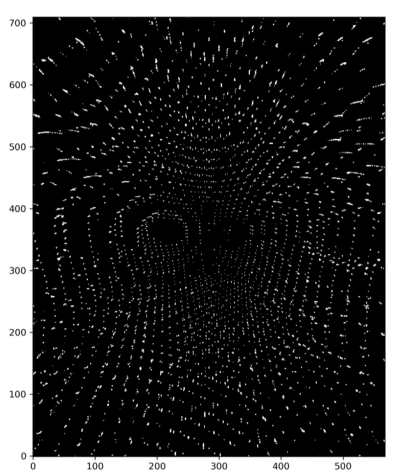

(a) Simulated output for a given (b) Emulated output for the correRDX configuration.

sponding RDX configuration.

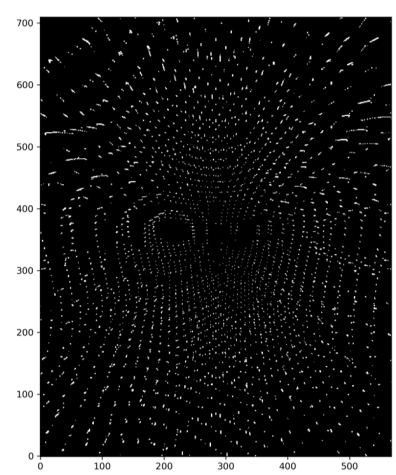

Fig. 5. Diffraction output for RDX from (a) simulation and (b) emulation.

to the simulations to determine the best match of diffraction patterns between simulation and experiment: thus indicating an optimal set of parameters. Producing enough simulations to determine optimal parameters using diffraction images is computationally expensive, especially since specific connections between strength model parameters and diffraction images are currently unknown. To explore a large enough space of possible parameters and solutions, emulation of the diffraction patterns is introduced. However, differences between emulation and simulation also need to evaluated. Comparing emulated patterns versus simulated patterns provides an opportunity to evaluate visualization tools without the need for an experimental comparison.

The emulator was trained using 1000 different simulations of a single plate impact experiment on $\alpha$-RDX. The strength model parameters were varied randomly for each simulation. The different parameter sets produced slightly different diffraction patterns for each simulation. Once the emulator was trained on these simulations, the emulator was also evaluated at the same 1000 input parameters, producing a similar set of diffraction patterns.

Diffraction output from one such set of parameters from both simulation and emulation is presented in Figure 5. As can be seen from this figure, the Bragg spots are sparse and often spread over only a few pixels. Further, for comparative purposes, it is non-trivial for the domain experts and this will require specialized visualization tools for effective exploration of such 1000 pairs of images.

\section{A. Comparative Visualization of Spots}

For effective exploration of these set of images, we use the Cinema viewer [3]. Cinema is a widely used framework for image-based data exploration. If the data to be explored can be structured in the form of a simple comma separated values (CSV) format, then the Cinema-based viewer becomes useful for exploration. In our case, we can create CSV files where each row represents one RDX configuration and its corresponding image outputs from simulation and emulation. Using the Cinema-based visualization, the RDX configurations 


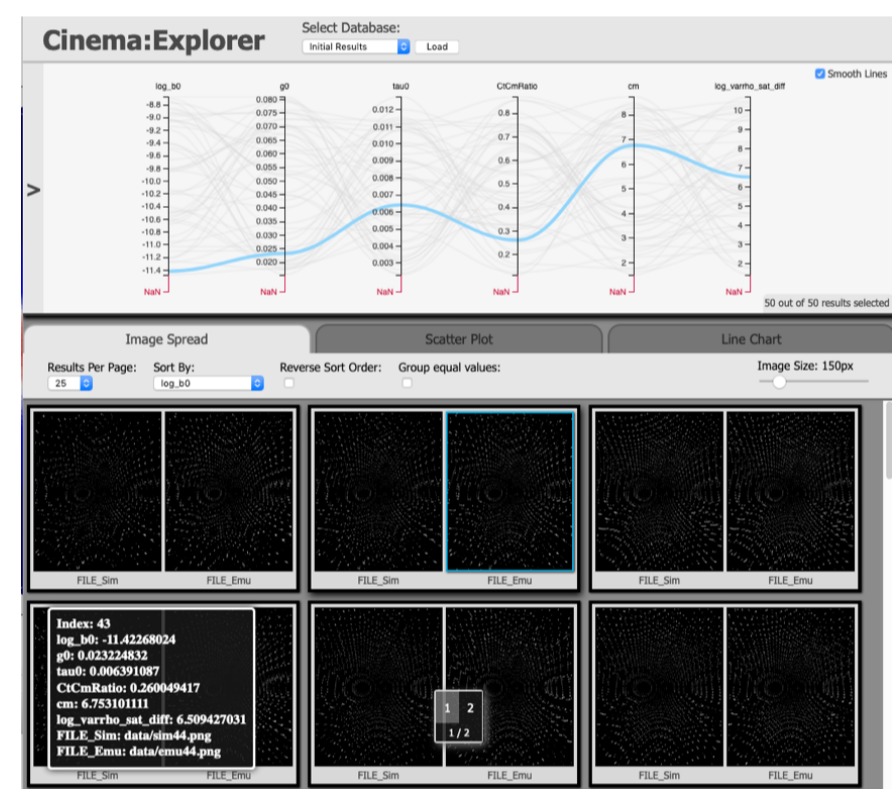

Fig. 6. Schematic representation of our proposed method using Voronoi tessellation.

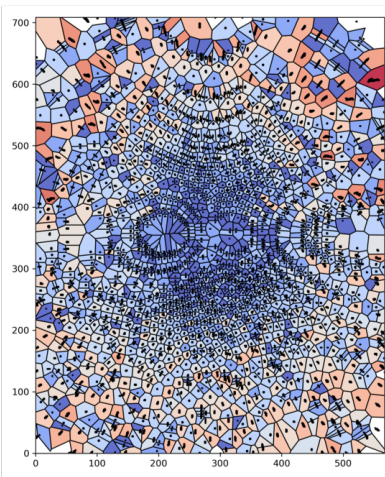

(a) Sim output.

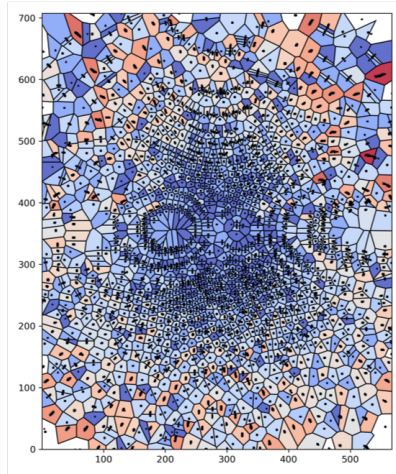

(b) Emulator output.
Fig. 7. Voronoi tessellation of space for a) simulation and b) emulation. Color corresponds to original spot size (blue small, red large).

will be shown at the top of the view panel (refer to Figure 6) using Parallel Coordinate Plots (PCP). Since Cinema supports comparative visualization by placing the image files side-by-side, user selected parameter combinations produce a corresponding image pair shown/highlighted at the bottom of the view panel. Using this setting, as shown in Figure 6 , exploration of the set of diffraction images with their corresponding parameter combinations, becomes tractable.

\section{B. Voronoi-based Visualization of Spots}

Given Figure 5, one of the primary tasks for the user is to understand the quality of emulation by comparing Figure 5(a) and Figure 5(b). The sparsity and similarity of the diffraction patterns overwhelm the subtle differences between the two images making the differences difficult to identify.

To alleviate this problem, we attempt to enhance the spots via Voronoi tessellation. Given an image, first the data is thresholded to assign 0 to the background and 1 to the pixels that contains a photon hit. This allows for separation of background from the foreground.

Next, we attempt to identify the individual spots. Each spot is defined by a collection of neighboring pixels that have high intensity (i.e., value of 1). To achieve this, we apply flood-fill based connected components algorithms on this thresholded images. Connected component algorithm identifies each spot as a cluster of pixels and assigns a unique identifier. At this stage, it also becomes possible to collect the different statistics regarding each spot, such as spot center and size etc. Now, instead of accounting for all the pixels with value 1, now only the spot centers can be used to represent the spots along with the size information. This offers an efficient data reduction and enables further comparative analysis.

After the spot centers and their corresponding sizes have been identified, the next goal is to provide an enhanced visualization such that simulated and emulated images can be compared. To achieve this, we use the spot centers as seed points on a 2D image plane and generate a Voronoi tessellationbased partition of the space. Basically, this tessellation assigns regions to every seed such that all the points within that region is closest to that seed point. Now, using a colormap, the size information of each spot center can be effectively depicted. An example of this is shown in Figure 7. Compared with Figure 5, now the spots are more pronounced and their size information is also much easily discernible via visual inspection. Further, now the comparison process becomes much easier and the difference between Figure 7(a) and Figure 7(b) can be much better visualized. After transforming all the raw diffraction images to this Voronoi space, Cinema-based exploration can be performed as shown in Figure 8.

\section{Difference-based Visualization of Spots}

As an alternative to the visual inspection of the simulated and emulated images, another method is to take the difference between the two images first and then visualize this difference. Although this is an attractive option, but there are certain problems that make this non-trivial. The number spots in the simulated and emulated images can be substantially different. Then, finding an association between these two sets of points become problematic. If we only want to find out the exact overlaps between points, then there will be many points that do not overlap, but still may be very close. The existing solution used by the domain scientists follow this idea of exact overlaps and is shown in Figure 9(a). In this figure, if there is an exact overlap, that pixel is colored in black. When the exact overlap does not happen, then if one pixel has a spot in simulated image, but not in emulated image, then that pixel is assigned color cyan. Finally, orange color is assigned to a pixel that has a spot in emulated data but not in simulated output. As can be seen, this pixel-wise coloring is not very helpful unless users zoom into the difference image.

For the diffraction application, it is not necessary that the simulated and emulated spots should overlap exactly for the emulated spots to be labeled as good quality. Similarly, if 


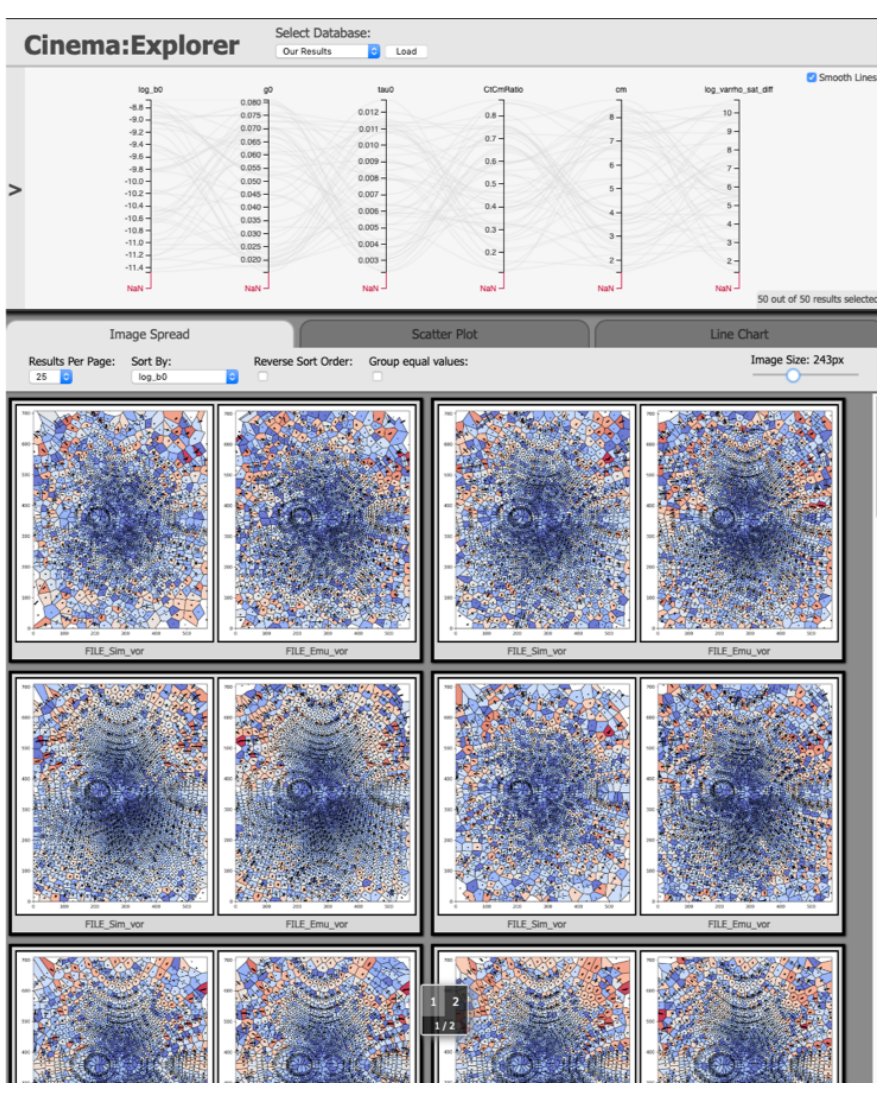

Fig. 8. Schematic representation of our proposed method using Voronoi tessellation.

the spots do not overlap, instead of labeling them "wrong", the inaccuracy should be computed based on the distance of the corresponding spots. For example, if the spots only differ by few pixels, they should be penalized less than if they differ by a much bigger distance. In this work, we take up this approach. In our method, we first compute the spot centers for simulated and emulated spots and then attempt to assign a correspondence across them based on their locations. For each emulated spot center, we find its closet match from the simulated spot center and then this simulated spot center is removed from the choice pool. This process starts from the sim-emu pair that has the minimum distance (likely overlapping points). While we try to assign one simulated spot center to an emulated spot center, we record the distances as an attribute of the emulated spot centers. Next, we can now create a Voronoi tessellation of the space now using the emulated spot centers and each region is now colored to reflect the distances from the simulated data. It is quite often possible that emulated result produces more spots. After the correspondence is established, since it is a one-to-one process, the extra emulated spots will be "orphan". For these orphan points, we use a different color (green) outside of the previously used colormap (cool-warm) to indicate the regions.

The result of this visualization is shown in Figure 9. Compared with the existing method of visualization as shown in Figure 9(a), our proposed visualization (as shown in Figure

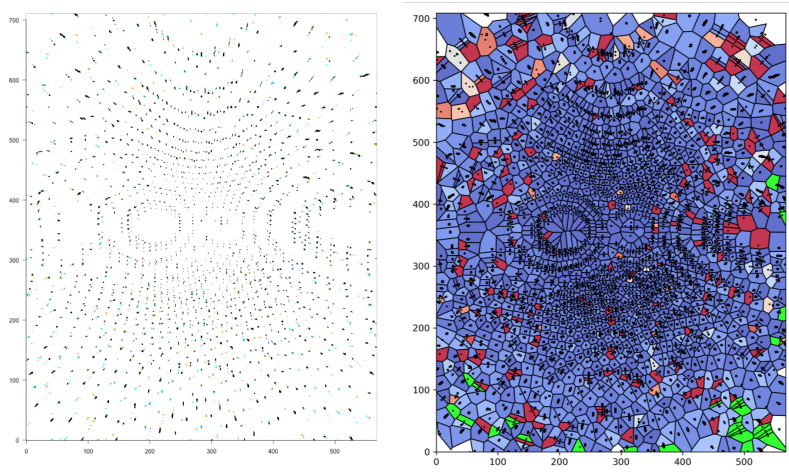

(a) Existing difference visualiza- (b) Our proposed difference visution.

alization.

Fig. 9. Compared to the existing method of visualization (as shown in a), our method enhances the difference visualization (as shown in b)

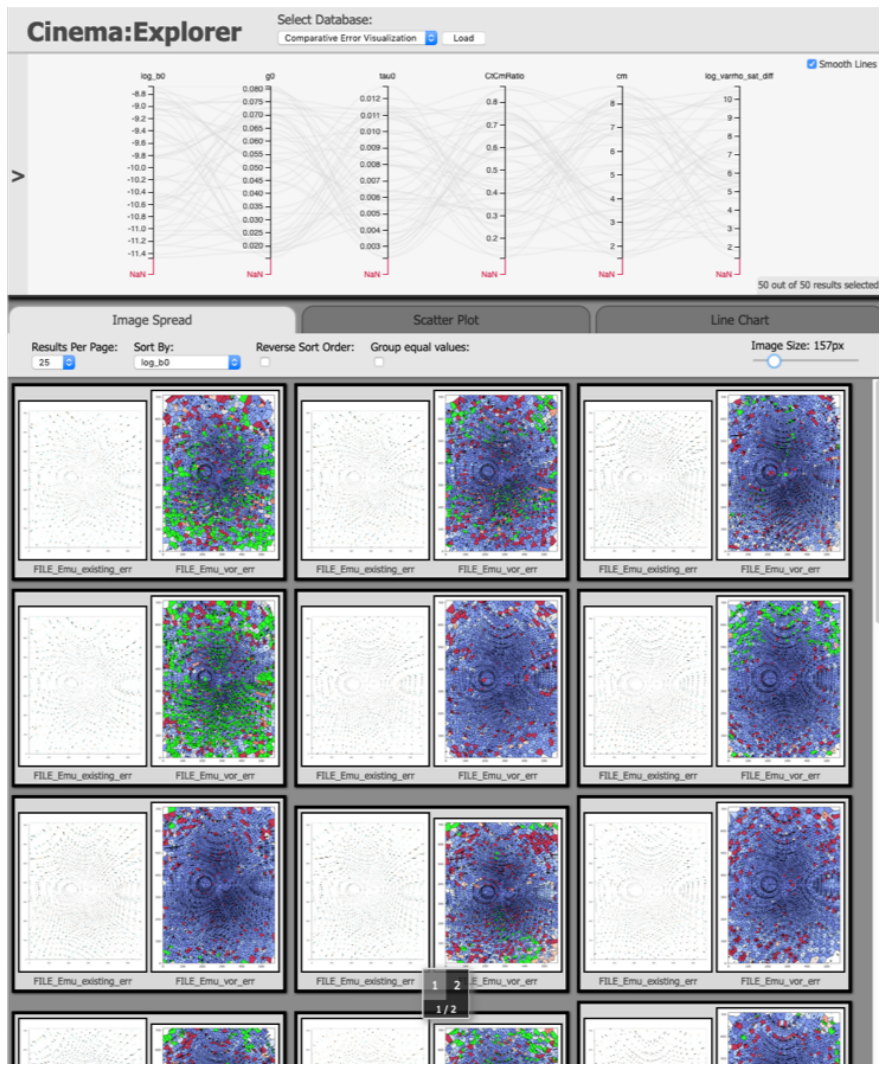

Fig. 10. Schematic representation of our proposed method using Voronoi tessellation.

9(b)) readily enables the users to spot the regions with high error (regions with red) as well as the regions where extra emulated spots were produced (regions with green). We can also used the Cinema viewer for exploring the difference images (as shown in Figure 10).

\section{EXPert FEedback AND Future Work}

The new visualization using Voronoi tessellation was able to preserve many of the spatial features of the original diffraction 
image while also encoding characteristics of the image in a less sparse format. This new visualization (Fig. 7) with Cinema: Explorer (Fig. 8) enabled a better overview than the original images since the spot size (color) and density of spots (Voronoi cell size) where encoded using different visual cues (size and color). However, this original manifestation in modifying how the data was visualized did little to assist in evaluating the differences between two different diffraction patterns.

The previous method for comparing two different diffraction images (pixel color based on overlap) was easy to interpret but difficult to inspect due to the sparsity of the data (Fig. 9(a)). Encoding the distance between matched pixels with color enabled a quick comparison of different diffraction images without needing to zoom in to inspect different spots on a pixel-by-pixel basis. This visualization scheme along with Cinema: Explorer enabled a rapid and accurate way to compare differences between the data. For example in Fig. 10 , it is obvious that the two diffraction patterns compared in row 2, column 1 are quite different in the number of diffraction spots produced between emulation and simulation. Others show better overall agreement between two images with some indicating clusters or regions of disagreement. Most of this inspection can be done quickly and using the Cinema filtering tools, groups of input parameter sets can be quickly inspected. Using the previous technique of coloring individual pixels based on overlap, evaluating hundreds or thousands of image comparisons would be nearly impossible. Using the visualization tools developed here shortens analysis time significantly while also illustrating important characteristics in the data in an obvious way.

With more fine-tuning of the visualization tools, some numerical metrics could also be computed using the parameters designated for visualization. For example, computing histograms of the percentage of cells containing certain colors (whether pertaining to spot size in a single image or nearest neighbor in a comparison image) could enable filters on the output. These types of filters could be useful for identifying patterns in input parameters based on outputs containing unique subsets of features. These kinds of tools are and will be useful for understanding patterns and characteristics associated with evaluating single crystal X-ray diffraction pattern images.

\section{CONCLUSION}

Enhancement of the visualization of diffraction patterns is an important topic to the scientists since it can reveal the crystalline structures of molecules. The existing methods do not readily provide the information that the scientists are looking for. Another task of this work is understand the differences between two sets of diffraction patterns when one is used as a surrogate model for the other. Since the diffraction patterns (Bragg spots) are often a few pixels wide and are sparse, in this work, we used Voronoi tessellation for enhancing the visualization. Compared with the existing methods, now our proposed work clearly highlights the regions of interest in the images. This further enables easy identification spot differences when surrogate models are used. User feedback shows the usefulness of our method.

\section{ACKNOWLEDGMENT}

We wish to acknowledge support from the U.S. Department of Energy's Advanced Simulation and Computing program, in particular the ATDM/ML and ASC/PEM projects at Los Alamos National Laboratory. We would also like to thank the anonymous reviewers for their suggestions in improving this manuscript. This work was done in Los Alamos National Laboratory.

\section{REFERENCES}

[1] Luscher, D.J., Addessio, F.L., Cawkwell, M.J., Ramos, K.J., 2017. A Dislocation Density-based Continuum Model of the Anisotropic Shock Response of Single Crystal $\alpha$-cyclotrimethylene Trinitramine. J. Mech. Phys. Solids 98, 63-86

[2] Burton, D.E., 1992. FLAG, a Multi-Dimensional, Multiple Mesh, Adaptive Free-Lagrange, Hydrodynamics Code. In: Nuclear Explosives Code Developers Conference.

[3] James Ahrens, Sébastien Jourdain, Patrick O'Leary, John Patchett, David H. Rogers, and Mark Petersen. An image-based approach to extreme scale in situ visualization and analysis. In Proceedings of the International Conference for High Performance Computing, Networking, Storage and Analysis (SC '14). IEEE Press, Piscataway, NJ, USA, 424434, 2014

[4] D. Francom, D. Walters, J. Barber, D. Luscher, E. Lawrence, A. Biswas, C.M. Biwer, D. Banesh, J. Lazarz C. Bolme, J. Ahrens, Emulation of Xray Diffraction from Dynamic Compression Simulations, (In preparation, 2019)

[5] David J.; Biswas, Ayan; Lawrence, Earl Christopher; Francom,Devin Craig; Luscher, Darby Jon; Fredenburg, David A.; Moran, KellyRenee; Sweeney, Christine Marie; Sandberg, Richard L.; Bolme, CynthiaAnne; Ahrens, James Paul, Bayesian calibration of strength parameters using hydrocodesimulations of symmetric impact shock experiments of Al5083Walters, Journal of Applied Physics, 2018

[6] A. Biswas, K. R. Moran, E. Lawrence and J. Ahrens, "Visualization of Uncertainty for Computationally Intensive Simulations Using High Fidelity Emulators," 2018 IEEE Scientific Visualization Conference (SciVis), Berlin, Germany, 2018, pp. 4 